\title{
Research on Mobile Chassis System of Live Working Robot
}

\author{
W. Song \& X.L. Zhou \\ State Grid Chongqing Electric Power CO. Electric Power Research Institute, Chongqing 404100, China
}

P.J. Si

Henan Polytechnic University, Jiaozuo 454003, China

C.J. Wang

Shenzhen Institute of Advanced Smart Technology, Shenzhen 518055, China

\begin{abstract}
With the progress of science and technology, more and more people pay attention to the research of robot technology. Comparison of biped robot, chassis wheeled mobile robot in moving speed, stability, application scope, carrying capacity has obvious advantages on, and especially the application field of wheeled mobile robot is more common in the field of service robot, robot for inspection and detection robot. Robot can durable and stable operation, is inseparable from the good mechanical system, this paper mainly for wheeled mobile robot motion analysis, design a can be used in the narrow space of the full range of mobile robot moving chassis system, and based on the idea of modular and system design, to meet the functional requirements of the mobile robot.
\end{abstract}

KEYWORD: Chassis wheeled mobile robot; Inspection and detection robot

\section{INTRODUCTION}

Mobile robot is a machine device can automatically perform the task. It can receive the human command and run pre-set procedures, can also according to set the artificial intelligence technology standard of principle to action, robot task is to assist or replace human complex, dangerous and repetitive work. In the world, the concept of robots has been gradually tend to be consistent, that the robot is a machine by the power and control their own ability to achieve the variousm functions. The United Nations Organization for standardization adopted Robot Institute of America defines a robot: "a be programming and multi-function, used to transport materials, spare parts, tools to operate the machine; or to perform different tasks and can change and action programming specialized system." And the definition of the robot in our country is: "the robot is a kind of automatic machines, the difference is this robot has some similar man-made biological intelligence capabilities, such as perception, planning ability, motor ability and synergy is a highly flexible automation machine". In this paper, the mobile chassis system of live working car is mainly used in the power distribution network maintenance, of course, can also be used in other dangerous operation environment.

\section{RESEARCH STATUS OF MOBILE ROBOT}

In the early sixties of the 20th century, western developed countries began the study of intelligent mobile robot, early mobile robot mainly for ground mobile robot, on behalf of the robot are mainly: Shakey Stanford Research Institute developed and French hilare research program to develop a robot hilare, Moravec developed Stanford cart and so on.

Mobile robot Shakey is the first mobile robot using artificial intelligence. Have certain artificial intelligence to autonomous perception, environment modeling, behavior planning and executing task, it has a camera and a feeling of detector, the airborne processor through a wireless signal with a host computer communication, visual processing and mission planning is completed by the host computer, motor control is by the onboard processor according to the main computer return signal to achieve (Kargov A. et al, 2008). AGV automatic guided vehicle is to equip with electromagnetic or optical automatic guiding device, can travel along the provisions of the guide lines, with safety protection and all kinds of transplanting function transport vehicle, and belongs to a kind of wheeled mobile robot. 


\subsection{Mobile robot navigation technology}

Robot navigation technology is one of the core technologies of mobile robot, and it is also the basic function of the mobile robot. It can make the mobile robot through the sensor to perceive the surrounding environment information and its own working state, in the absence of human intervention, to achieve the desired goal. At present, the mobile robot navigation methods include: several magnetic navigation, inertial navigation, GPS navigation, visual navigation, the magnetic navigation is mainly used in the automatic guided vehicle. Visual navigation is through the installation of the camera on a mobile robot to complete the obstacles and road sign detection and recognition, is a development direction of robot navigation technology in the future, but in image recognition, and other key technical field, there are still larger problems (Lauria M. et al, 2006).

\subsection{Kinetic analysis of mobile robot chassis}

In the mobile robot and wheeled robot compared to double foot robot control is simple, has better stability, speed faster, compared crawler robot noise and ride comfort better. Therefore, wheeled robot has good comprehensive properties, in addition, wheel mechanism is simple, easy disassembly, easy to maintenance and repair, based on the product design of the site is more suitable for, for mobile robot wheel layout, many scholars have done a lot of research, and general layout is divided for three wheel configuration and four wheel configuration.

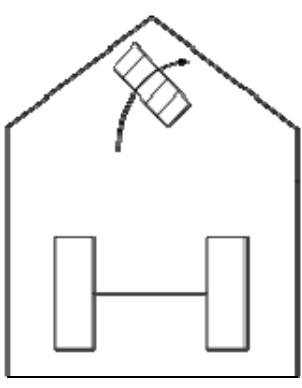

(a)

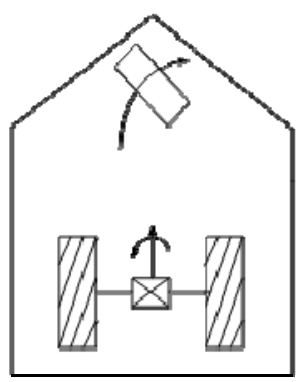

(c)

Figure 2 .Three wheeled WMR model
The four round of the layout as shown in the figure, generally can be divided into the rear wheel drive (a), front drive wheel (b) and the left and right wheel diagonal drive (c) three. The four round of layout compared three layout better stability, for the first drive, due to the driving of front wheel drive vehicles should not only be responsible and responsible for the vehicle, the front axle load is heavy, which makes the front wheel drive vehicles in the turn the front focus because of inertia moved forward, easy to break through the front grip, and rear wheel the lack of power, will be driven by the understeer; while the rear wheel drive rear wheel drive can be responsible, responsible to work, so when the vehicle steering is more responsive, better start acceleration, and vehicle speed at the start, or when climbing due to gravity after the shift, as the rear wheel drive wheel can effectively improve the tire grip. For vehicle starting, acceleration and climbing action, improve vehicle stability and comfort, so this The use of the rear wheel independent drive; the last configuration, if the wheels are used in all directions, it can make the vehicle with the ability to move horizontally.
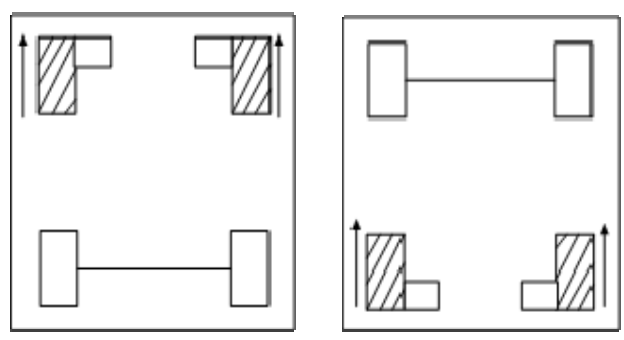

(a)

(b)

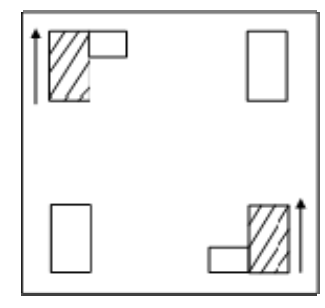

(c)

Figure 3. Four round WMR model

To sum up, we should give high priority to the use of four wheel layout, the rear wheel drive, for four wheel structure uniformly reliability assurance by assigning the buffering suspension system to achieve, and the front wheels with omnidirectional wheel can make up for the four wheel arrangement robot steering motion are needed in order to achieve the shortcomings of certain forward stroke and realize the function of turning on the spot. The electric operation vehicle mobile robot using the overall structure of the model diagram as shown in Figure 5, which represents 1 mobile chassis in this paper. 


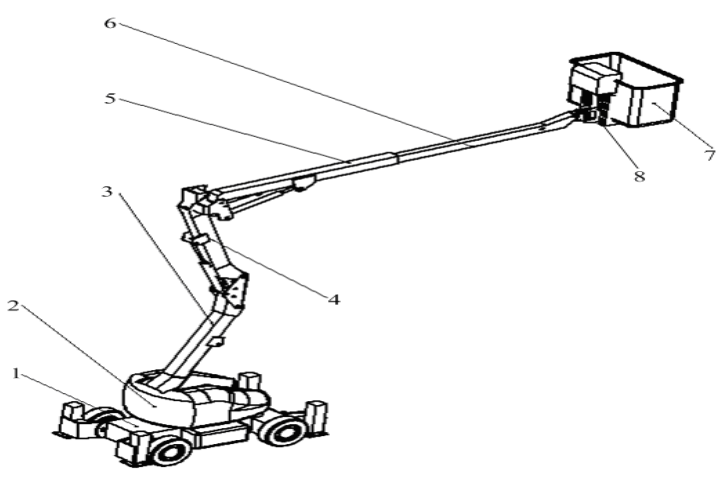

Figure 4. Overall structure of mobile robot

The two wheel drive, two wheel steering, maximum speed $7 \mathrm{~km} / \mathrm{h}$, maximum climbing slope $20 \%$, this decline cannot stop the slope and the slope on the machine, start walking; two brake - standard, automatic driving hydraulic tire using the standard release, spring applied brakes, and can make the aerial platform stop at $20 \%$ slope fall, hydraulic support leg leveling mechanism, can adapt to various ground conditions, to ensure the normal work of the working platform; part of the table, a 355 degree rotation of a carriage lockpin hole, a drive unit imported DC electric machine, powerful and reliable, power battery - battery, 42Ah, control system by the ground controller integrated all debugging function and arm action associated with, including the platform control handle. Machines and components are calibrated by the control system to process. All valve and controller was adopted to control the system and diagnosis system being controlled by the system self diagnosis occurs in the system and connected with the system all the components of the main types of errors, the main fault through centralized meter blinks indicating lamp and a buzzer alarm. The structure model of the chassis system is shown in figure 5:

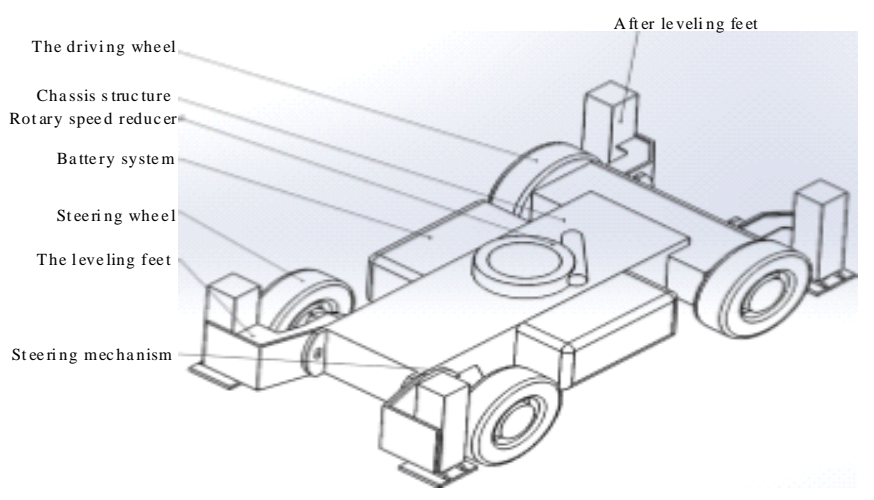

Fig.5 Chassis system of live working vehicle
Mobile robot control system based on the task of the robot to complete the task of the robot and the robot from the sensor feedback signal to control the robot to complete the task of the robot. The control system has the function of the robot, the robot's body structure and the control mode of the robot (Muir, P. and C. 1987).

Robot control system to meet the general requirements of a few basic requirements:

(1) The miniaturization, lightweight, standardization and modularization of the control system. The robot control system is placed on the robot, in order to facilitate the installation and links, requirements of control system as far as possible miniaturization; and control system as much as possible light. Only in this way can reduce the load of the machine, reduce the power consumption of the system; in addition, for the daily maintenance of the system, with good scalability, standardization system as much as possible and modularization;

(2) With good reliability. Squid interference signal will affect the normal operation of the robot, so to consider the allocation of hardware and software tasks and select the grounding, isolation, shielding, and other aspects of the factors;

(3) The system has a good stability. Stability is the basic requirement of the control system, and the basic problems of the most important task of the robot is to achieve stable walking according to the needs of mobile robot control system design, combined with the system function and characteristics of robot, based on the idea of modular design, the machine control system overall scheme, as shown in Figure 6.

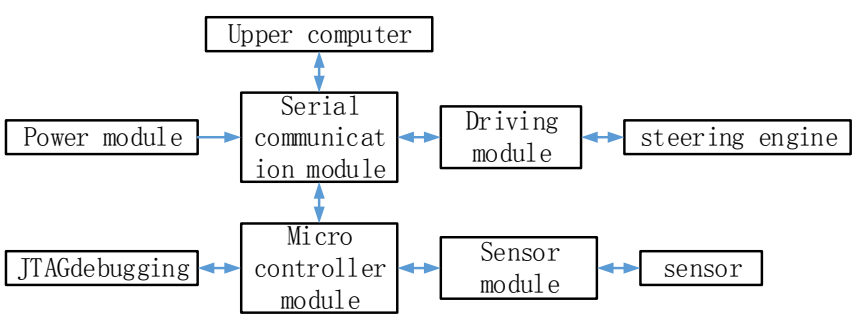

Fig. 6 chassis control system

\section{SUMMARY}

From the practical point of view, this paper presents the design method of the mobile chassis motion control system of the intelligent robot for live working vehicle. As follows: according to the characteristics and application background of the mobile robot, select the appropriate parts of the system, from two aspects of the structure and function of the complete motion control system design, in order to improve 
the ability of mobile robot outdoor sports, control algorithm based on the robot dynamic model design of stability, this algorithm can ensure the robot trajectory tracking performance, the electric operation vehicle chassis motion control system not only has the engineering practicability, and can serve as an experimental platform, tightly control algorithm research and improve the intelligent level of mobile chassis system of outdoor work.

\section{REFERENCES}

Kargov, A., et al., Development of an anthropomorphic hand for a mobile assistive $\operatorname{robot}[\mathrm{J}]$, in Rehabilitation Robots,2005.ICORR 2005.9th International Conference on.2008.p.426-431.

Lauria, M., et al., Design and Contronl of a Four Steered Wheelled Mobile Robot[J], in IEEE Industrial Electronics, IECON 2006-32nd Annual Conference on.2006.p.40204025.

Muir, P. and C. Neuman, Kinematic modeling for feedback control of an omnidirectional wheeled mobile robots[J], in Robotics and automation.Proceedings.1987 IEEE International Conference on.1987.p.1772-1778. 